\title{
Possibility of enhancing the dimensional stability of jelutong (Dyera costulata) wood using glyoxalated alkali lignin-phenolic resin as bulking agent
}

\begin{abstract}
The utilization of low molecular weight phenol formaldehyde (LmwPF) resin as bulking agent to enhance the dimensional stability of wood brought some disadvantages, such as phenol is derived from non-renewable petrochemicals while formaldehyde is a known carcinogen. Hence, the possibility of using bulking agent made of glyoxalated alkali lignin (GL) incorporated in LmwPF resin to enhance the dimensional stability of wood was investigated. FT-IR spectroscopy showed that polymerization of GL and LmwPF resin was accomplished via the formation of methyl ether $(\mathrm{CH} 2 \mathrm{OCH} 2)$ bridge. Small amount of crosslinked polymer network was accomplished via the formation of methylene $(\mathrm{CH} 2)$ bridge. Oven dried jelutong wood was evacuated under vacuum followed by soaking in 15, 20 and $25 \%$ concentrations of glyoxalated alkali lignin-low molecular weight phenol formaldehyde (GL-LmwPF) (67\% solid of GL: $33 \%$ solid of LmwPF based on the total solute content) and LmwPF resins, respectively at ambient temperature for $24 \mathrm{~h}$. The impregnated wood was then cured at $190{ }^{\circ} \mathrm{C}$ for $30 \mathrm{~min}$. The weight percent gain (WPG) and dimensional stability in terms of antiswelling efficiency (ASE), moisture excluding efficiency (MEE) and water absorption (WA) as well as leachability of bulking agents were determined and compared with untreated wood and wood treated solely with LmwPF resin. The WPG of GL-LmwPF treated wood was lower than LmwPF treated wood. GL-LmwPF treated wood exhibited positive ASE, but the values were lower compared to LmwPF treated wood. The MEE and WA of GL-LmwPF treated wood were also inferior to LmwPF treated wood and untreated wood. GL-LmwPF resin was leached out from the treated wood whereas no leaching was found for LmwPF treated wood after three leaching cycles in distilled water. The formaldehyde release of GL-LmwPF resin treated wood was $25.76 \%$ less than of wood treated with LmwPF resin. Wood treated with $25 \%$ GL-LmwPF resin yielded highest ASE value compared to 15 and $20 \%$ GL-LmwPF treated wood. Hence, wood treated with $25 \%$ GL-LmwPF resin together with external coatings could be used in several end applications such as parquet flooring, paneling and furniture component.
\end{abstract}

